Background Buruli ulcer is one of the neglected tropical diseases. It is a chronic, debilitating, necrotising disease of the skin and soft tissue caused by Mycobacterium ulcerans. Most times, the pattern of presentation is neglected by the infected because it is regarded as a disease of the poor who have little or no access to healthcare. Living in rural often inaccessible areas and suffering from a triad of ignorance, stigma and poverty, this poor population fails to present early to a hospital.

Methods A retrospective review of patients who accessed care at the infectious disease clinic of Nnewi Diocesan Hospital, Nnewi, Southeast Nigeria, between 1 January to 31 December 2017. To achieve a complete inference, the results of laboratory wound swab culture of all patients were collated and matched with the clinical presentation. All cultures were done by a trained scientist of the German Leprosy and TB Relief Association (GLRA).

Results Review of data showed a total of 10120 patients of which 6402 were outpatients and 3718 were inpatients; they were between 1 and 86 years of age. There were 60 cases of limb ulcers of which wound swab culture was done. Fifty-four (54) were diabetic foot ulcers while five (5) were venous ulcers. Acid-fast bacilli were detected with Ziehl-Neelsen staining in one specimen and confirmed by the reference center.

Conclusion Most of the Buruli ulcer patients are found incidentally following late presentation at hospitals with a questionable ulcer/wound with a high index of suspicion on clinical examination. If Buruli ulcer is to be eradicated, an intensive rural epidemiological identification programme must be implemented to isolate the infected. The vicious cycle of ignorance, stigma and poverty needs to be broken by massive awareness and education campaigns.

\section{PO 8372 CULTURE-FREE APPROACHES FOR THE DIAGNOSIS AND MANAGEMENT OF PATIENTS WITH RIFAMPICIN RESISTANT TUBERCULOSIS: THE DIAMA PROJECT}

\begin{abstract}
${ }^{1}$ Faridath Massou*, 'Dissou Affolabi, ${ }^{2}$ Corinne Merle, ${ }^{3}$ Gemeda Abebe, ${ }^{4}$ Oumou Bah Sow, ${ }^{5}$ Bassirou Diarra, ${ }^{6}$ Osman El Tayeb, ${ }^{7}$ Ayou Gaye Diallo, ${ }^{8}$ Michel Kaswa, ${ }^{9}$ Jean Claude Ngabonziza Semuto, ${ }^{10}$ Melissa Sander, ${ }^{11}$ Philip Supply, ${ }^{12}$ Bouke De Jong. ${ }^{1}$ Supranational Reference Laboratory of Mycobacteria, Cotonou, Benin; ${ }^{2}$ Special Programme for Research and Training in Tropical Diseases, World Health Organization (WHO/TDR), Geneva, Switzerland; ${ }^{3}$ Jimma University, Jimma, Ethiopia; ${ }^{4}$ Service de Pneumophtisiologie, Guinea, Conakry; ${ }^{5}$ Université des Sciences, des Techniques et des Technologies de Bamako, SEREFO, Mali, Bamako; ${ }^{6}$ Damian Foundation, Ibadan, Nigeria; ${ }^{7}$ Université Cheikh Anta Diop, Senegal, Dakar; ${ }^{8}$ Institut National de Recherche Biomédicale (INRB), Kinshasa, DRC; ${ }^{9}$ Rwanda Biomedical Center (RBC), Kigali, Rwanda; ${ }^{10}$ The Tuberculosis Reference Laboratory Bamenda, Cameroon; ${ }^{11}$ Genoscreen, France, Lille; ${ }^{12}$ Institute of Tropical Medicine (ITM), Belgium, Antwerp
\end{abstract}

\subsection{6/bmjgh-2019-EDC.77}

Background Recent advances in molecular diagnostics, especially the Xpert MTB/Rif test, have reduced the time to diagnose rifampicin resistant tuberculosis (RR-TB). However, with this test only rifampicin resistance is diagnosed, leading to presumptive diagnosis of resistance to isoniazid and maybe other drugs. In addition, culture on monthly sputum samples is currently recommended by the World Health Organization (WHO) for follow-up of RR-TB patients under treatment. Unfortunately, culture is often not locally available, and samples need to be shipped from field to culture laboratories. The associated transport delays lead to high rates of contamination and false-negative culture, particularly in laboratories in low-resource settings. Many gaps for the diagnosis and management of RR-TB patients still need to be addressed and the DIAMA project (Diagnostics for multidrug-resistant tuberculosis in Africa) aims to address some of them.

Methods The TB Supranational Reference Laboratory of Benin leads a consortium of 11 partners involved in multiple-drug resistant TB care in Africa. The DIAMA project will explore the feasibility and accuracy of: i) diagnosing $\mathrm{TB}$ resistance to first and second line drugs through novel molecular multiplex assays developed by the company Genoscreen; ii) setting-up alternative culture-free approaches for the monitoring of patients' response to rifamipcin-resistant treatment; iii) piloting whether the implementation of software by Data2Care Technologies for real-time monitoring of molecular test results can reduce delays between diagnosis and treatment of RR-TB patients. This project is funded by EDCTP for a period of five years.

Conclusion Together, these advances could dramatically improve the currently dismal prognosis of multiple-drug resistant $\mathrm{TB}$ in health systems in resource-poor settings. Through this presentation, we will share the background information, the design of this project and its progress.

\section{PO 8381 REGULATING CLINICAL TRIALS DURING AN EBOLA EMERGENCY: THE LIBERIAN EXPERIENCE}

${ }^{1}$ Theophilus Ndorbor*, 'David Sumo, 'Juwe D Kercula, ${ }^{2}$ Ezekiel F Hallie. 'Liberia Medicines and Health Products Regulatory Aurthority, Monrovia, Liberia; ${ }^{2}$ School of Pharmacy, University of Liberia, Monrovia, Liberia

\subsection{6/bmjgh-2019-EDC.78}

Background Effective clinical trials oversight is a major function of a fully functional national medical products regulatory system. However, exercising clinical trial oversight in a resourcelimited environment is challenging, in particular during an Ebola outbreak or health emergency. Until the devastating Ebola virus disease (EVD) outbreak in 2014, the Liberia Medicines and Health Products Regulatory Authority (LMHRA) had no capacity for effective clinical trial regulation. This presentation describes the main challenges encountered by LMHRA in regulating clinical trials in Liberia during the largest EVD outbreak that affected West Africa in 2014 and 2015.

Methods By carefully documenting activities during the EVD outbreak, interviewing key stakeholders, and discussions among the LMHRA clinical trial committee, key challenges observed during the outbreak were identified and documented.

Results Limited financial resources, lack of expertise in clinical trials, inaccurate and insufficient information about the functions of the LMHRA, poor coordination among key stakeholders, and the lack of a well-developed regulatory framework, adversely influenced the LMHRA clinical trial oversight performance during the EVD outbreak.

Conclusion It is true that several challenges need to be addressed when regulating a clinical trial in a limited-resource environment during any disease outbreak or international medical emergency. However, the importance of building local expertise in clinical trials through mentorship and training cannot be overemphasised. By taking advantage of grants from developmental partners, national medicines regulatory authorities in resource-limited environments can develop capacity for clinical research oversight. 